\title{
Chemical-Garden Formation, Morphology, and Composition. II. Chemical Gardens in Microgravity
}

\author{
Julyan H. E. Cartwright, ${ }^{*,+}$ Bruno Escribano, ${ }^{*,+}{ }^{\S}$ C. Ignacio Sainz-Díaz, ${ }^{*,+}$ and Louis S. Stodieck ${ }^{*,+}$ \\ ${ }^{\dagger}$ Instituto Andaluz de Ciencias de la Tierra, CSIC-Universidad de Granada, Facultad de Ciencias, E-18071 Granada, Spain \\ ${ }^{\ddagger}$ Department of Aerospace Engineering, BioServe Space Technologies, University of Colorado at Boulder, Boulder, \\ Colorado 80309-0429, United States
}

Supporting Information

ABSTRACT: We studied the growth of metal-ion silicate chemical gardens under Earth gravity $(1 \mathrm{~g})$ and microgravity $(\mu \mathrm{g})$ conditions. Identical sets of reaction chambers from an automated system (the Silicate Garden Habitat or SGHab) were used in both cases. The $\mu \mathrm{g}$ experiment was performed on board the International Space Station (ISS) within a temperature-controlled setup that provided still and video images of the experiment downlinked to the ground. Calcium chloride, manganese chloride, cobalt chloride, and nickel sulfate were used as seed salts in sodium silicate solutions of several concentrations. The formation and growth of osmotic envelopes and microtubes was much slower under $\mu \mathrm{g}$ conditions. In $1 \mathrm{~g}$, buoyancy forces caused tubes to grow upward, whereas a random orientation for tube growth was found under $\mu \mathrm{g}$ conditions.

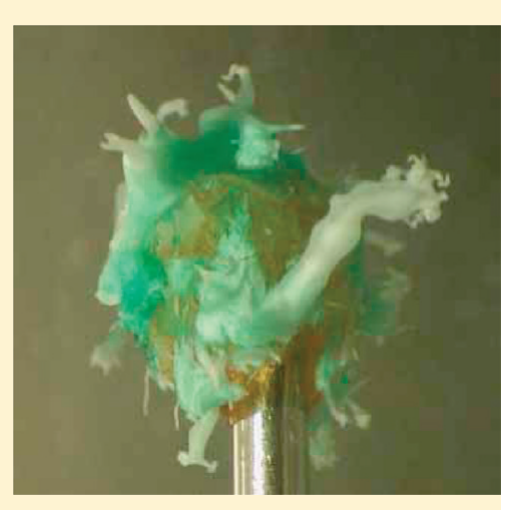

\section{INTRODUCTION}

Chemical gardens, or silicate gardens, grow when a solid of a metal-ion salt is placed into a sodium silicate solution. ${ }^{1}$ As the salt begins to dissolve in the silicate, it develops a colloidal semipermeable membrane of metal silicate. Osmotic pressure pulls water from the silicate into the enveloping membrane, further dissolving the salt. The membrane is consequently inflated, forming what we term an osmotic envelope, until it ruptures and expels a jet of metal-ion solution. This solution has a different $\mathrm{pH}$ from that of the external solution and the silicate precipitates forming a hollow tube through which the metal-ion solution continues to flow, pumped by the semipermeable membrane. The tube continues growing at its tip by accretion of metal-ion precipitate until the initial salt is depleted. Hydroxide ions that enter through the osmotic membrane react with the metal ions and precipitate on the inner surface of the tubes as metal hydroxides. The morphology of these silicate gardens depends on the evolution of the combination of forced convection driven by osmotic pressure through the semipermeable membrane and free convection due to buoyancy forces. ${ }^{1}$ Under normal gravity conditions, the tubes grow generally upward under a combination of osmotic pressure and buoyancy forces, because the metalion salt solution is less dense than the surrounding silicate, so it is of interest to find out how the absence of buoyancy forces under conditions of microgravity affects chemical-garden growth. Buoyancy forces giving rise to convection are proportional to gravity. When chemical gardens are grown in microgravity, the buoyancy effect is diminished correspondingly and the tubes, driven by forced convection alone, would be expected to grow in arbitrary directions.

Although chemical-garden growth is a long-studied phenomenon, ${ }^{2-4}$ it is not yet well understood with regard to the physical and chemical variables that control the morphology of these microtubes. Gaining a greater insight into these processes would potentially be of value for materials sciences. Furthermore, the phenomenon is of interest for educational purposes, since in addition to having an attractive visual impact with students, chemical-garden growth is an excellent system in which to study the chemical processes of dissolution, precipitation, and crystallization interconnected with physical phenomena of fluid dynamics and osmosis. Previously, we explored salts with cations from the same group in the periodic table, that of group 2 of the alkaline-earth cations, and observed similar behavior but different growth rates. ${ }^{5}$ We have also studied the behavior of salts within the same period, period 4 , in the periodic table: ${ }^{6}$ a study that we complete in this work with microgravity experiments.

A sole experiment on chemical-garden growth in microgravity, which flew aboard the shuttle mission STS-55 in 1993, has been reported in the literature by Jones and Walter. ${ }^{7}$ There was an earlier experiment than Jones and Walter's flown on STS-47 in 1992, but inexplicably, the researchers reported only on the

Received: October 18, 2010

Revised: January 9, 2011 


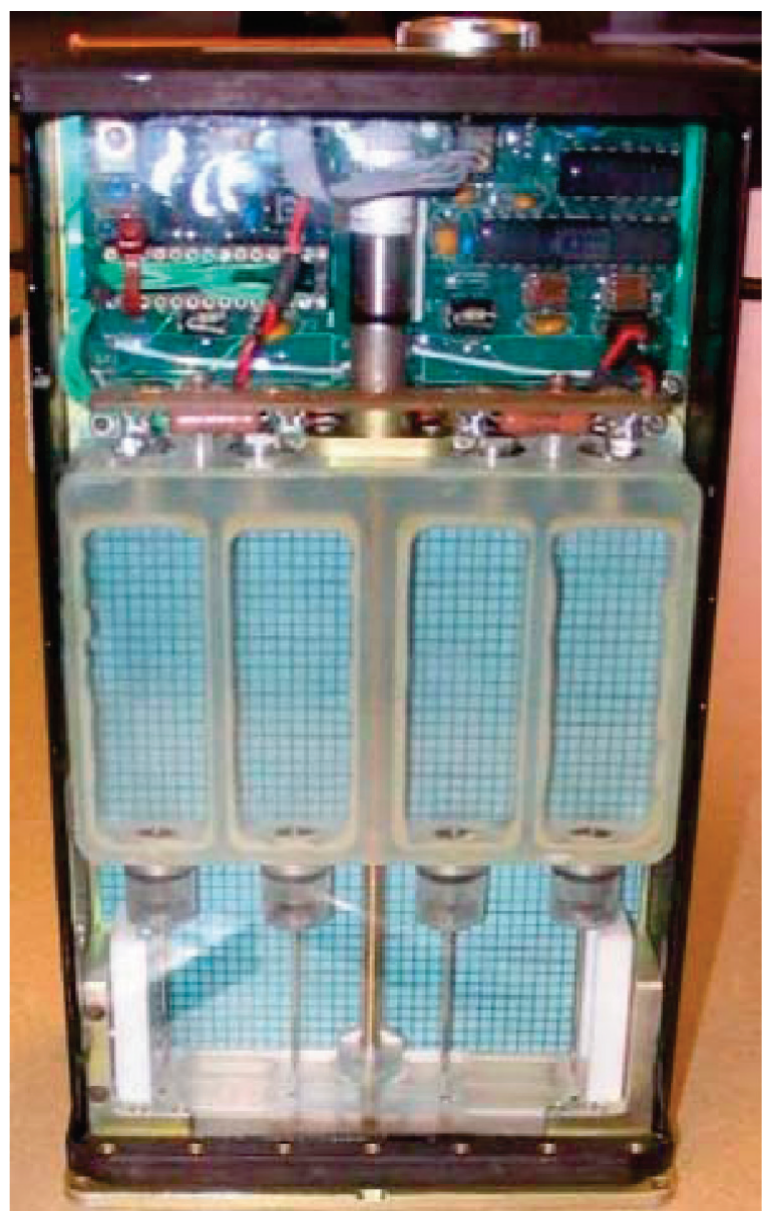

Figure 1. Experimental apparatus, the Silicate Garden Habitat (SGHab) developed by BioServe Space Technologies. The width is approximately $10 \mathrm{~cm}$ and the height $20 \mathrm{~cm}$.

details of the hardware flown and not on the experimental results they obtained, although tantalizingly they mentioned twisted tube growth in random directions and in some cases 'spiral growth. 8 Subsequent to Jones and Walter, the experimental setup reported on here was first flown aboard STS-107 in 2003. That experiment was lost when the shuttle Columbia broke up on reentry, as we recall in the dedication of this article to the astronauts who died.

Jones and Walter ${ }^{7}$ reported some unexpected results like reduced growth rates and plastic growth. However, questions remained on the physical aspects of this phenomenon. Following our preliminary experiments under Earth gravity, ${ }^{6}$ in this work we present experiments conducted on the the International Space Station, with some differences compared to the experimental setup of Jones and Walter and a different range of salts and silicate-solution concentrations, with two main aims: on one hand, to explore further the effect of microgravity on chemicalgarden growth and morphology, and on the other, to set up an approach for teaching high school and university students multidisciplinary science combining space science, engineering technology, chemistry, and fluid dynamics. ${ }^{9}$

\section{EXPERIMENTAL METHOD}

The reactor, the Silicate Garden Habitat or SGHab (Figure 1), was developed by BioServe Space Technologies and flown to the
International Space Station (ISS) on the space shuttle Endeavour. Two SGHabs were flown on separate missions, one in August 2007 (STS-118, ISS mission 13A.1) and a second in March 2008 (STS-123, ISS mission 1J/A). The SGHab was configured with eight chambers, four per side, prefilled with a given sodium silicate solution. Individual chambers were cuboidal in shape $(2.2 \mathrm{~cm} \times 5.9 \mathrm{~cm} \times 1.8 \mathrm{~cm}$ deep $)$ each with a total volume of $23 \mathrm{~mL}$ with transparent polycarbonate walls. During installation on the ISS, the experiment was assembled such that the four chambers on Side A could be viewed by the automated imaging system. Once the experiment on Side A had completed, the SGHab was turned around so that the experiment on Side B could be initiated and visualized.

Approximately $1 \mathrm{~g}$ of each of the different metal-ion salts was mixed with a small amount of epoxy glue and molded into a cylinder of about 5 $\mathrm{mm}$ in radius and $5 \mathrm{~mm}$ in height to be compatible with the reactor design. This preparation of the salt seed is different than that of our previous works, ${ }^{5,6}$ but it is valid for relative comparisons. The saltepoxy mixture was chosen instead of pure crystals to avoid anisotropy and to strongly fix the salt to the insertion rod. The salt cylinders were attached to stainless steel rods and held in place separated from the silicate-solution chamber. When the reactor was activated, the rods pushed the salts through a foil burst disk and a duckbill valve into the silicate-solution chamber. An automated camera and frame-capture system recorded the evolution of the reaction for several days and sent the resulting images back to Earth for analysis in real time.

The silicate concentrations were chosen to maximize the reaction velocity. Solutions were prepared from a commercial concentrated solution composed of $27 \% \mathrm{SiO}_{2}$ and $15 \% \mathrm{NaOH}$ by dilution with ultrapure water to concentrations varying from 0.6 to $6 \mathrm{M}$. The studied salts were $\mathrm{CaCl}_{2}, \mathrm{CoCl}_{2}, \mathrm{MnCl}_{2}$, and $\mathrm{NiSO}_{4}$. They were selected from our preliminary experiments under ground conditions with whole grains and pressed wafers. ${ }^{6}$ The cations of these salts belong to the same period, period 4, of the periodic table. This cation series shows different valence electron population with the sequence $\mathrm{Ca}<\mathrm{Mn}<\mathrm{Co}<\mathrm{Ni}$.

The SGHabs were returned to Earth on the Space Shuttle and the chemical-garden growths were recovered from the reaction chambers. Samples were extracted from the chambers and dried in air at $25{ }^{\circ} \mathrm{C}$. Morphology was studied using a FEI Quanta 400 environmental scanning electron microscope (ESEM) at high vacuum and room temperature. Chemical analysis of the different sections of the samples was performed in situ in the microscope using EDAX spectroscopy.

\section{RESULTS}

Buoyant Plume versus Forced Convection. When chemical gardens are grown on Earth, a semipermeable membrane is formed enveloping the seed salt as soon as it is immersed in the silicate solution. This membrane is inflated with water from the silicate solution forced into the interior of the membrane under osmotic pressure. As the membrane increases in volume, its walls become thinner, until a hole is formed through which a jet of metal-ion salt solution is expelled. Since the ejected metal-ion solution is lighter than the surrounding silicate, buoyancy forces become predominant and the tubes grow upward to the surface.

When we repeated the same experiments in space-in microgravity - we were removing the gravitational force and hence eliminating buoyancy. Under this condition, the reaction proceeded with forced convection driven by osmotic pressure alone and tubes were observed to grow out of the membrane in random directions. In Figure 2, we compare results from experiments performed on Earth and in microgravity with the four studied salts: $\mathrm{CaCl}_{2}, \mathrm{MnCl}_{2}, \mathrm{CoCl}_{2}$, and $\mathrm{NiSO}_{4}$. Silicate concentrations were selected from our preliminary work $^{6}$ to maximize the 


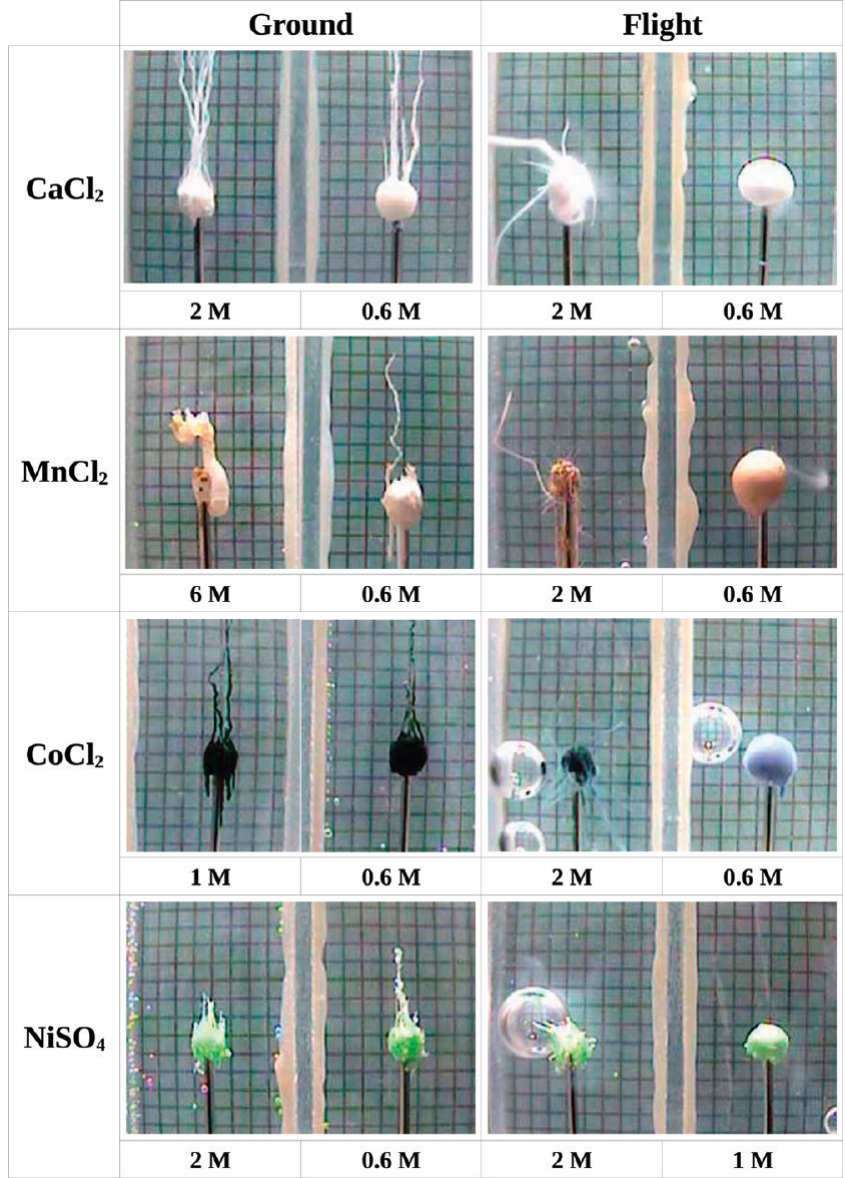

Figure 2. Comparison of similar experiments performed with and without gravity, at various sodium silicate concentrations. In ground experiments, tubes grow upward regardless of the location where the semipermeable membrane initially bursts. Flight experiments exhibit tubes that grow in random directions. Images from the Earth controls were taken after $8 \mathrm{~h}$ of growth, when the metal-ion salts were completely depleted. Images from the flight experiments were obtained in situ 5 days after initiation, after which time the SGHab had to be removed from the imaging unit owing to time-scheduling limitations on the International Space Station. The background grid lines are $2 \mathrm{~mm}$ apart.

growth, based on the report by Jones and Walter that silicate garden reactions in space would be much slower than on Earth. ${ }^{7}$

In all growth experiments with $6 \mathrm{M}$ silicate, the formation of many air bubbles, probably formed from gas trapped during wetting, ${ }^{2}$ prevented a clear observation of the results, especially in microgravity conditions. For the calcium in $6 \mathrm{M}$ silicate, under Earth gravity a transparent osmotic envelope is formed before the formation of tubes, according to our previous experiments. ${ }^{6}$ In experiments performed with 2 and $3 \mathrm{M}$ silicate, we could clearly see the difference in growth directions, growth being always vertical in the presence of gravity and random in its absence (Figure 2 and Figure 3). This same behavior also applies to the case of microscopic tubes, as we see in SEM imaging of calcium gardens grown in $3 \mathrm{M}$ silicate (Figure 4). The behavior of the ground experiment was similar for 2 and $0.6 \mathrm{M}$ silicate in calcium gardens, there being many narrow white tubes formed. The same salt immersed in $0.6 \mathrm{M}$ silicate in space did not grow any tubes after 5 days, whereas it took less than $8 \mathrm{~h}$ for the tubes to grow to the top of the chamber in the ground experiment. This lack of tube formation was observed in every experiment with lower

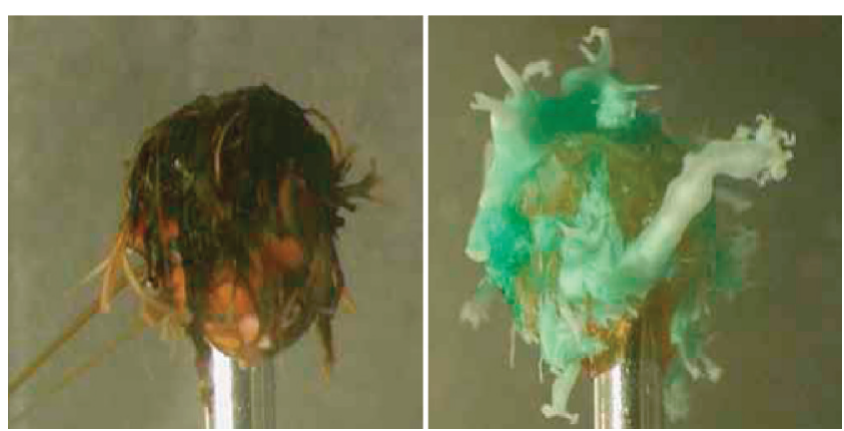

Figure 3. Images of flight experiments taken after returning to Earth and removing the silicate solution: (a) cobalt garden in $2 \mathrm{M}$ silicate; (b) nickel garden in $2 \mathrm{M}$ silicate. Pictures are approximately $1 \mathrm{~cm}^{2}$.

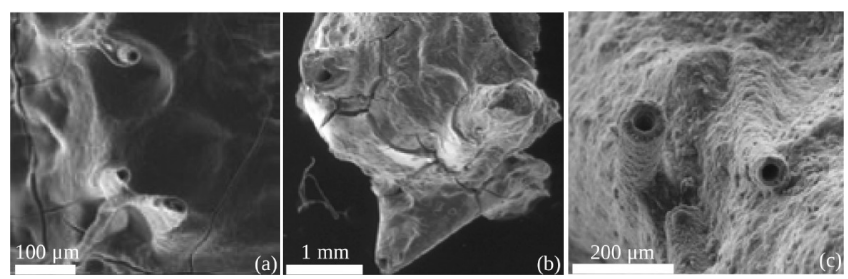

Figure 4. Micrographs of calcium tubes grown in $3 \mathrm{M}$ sodium silicate (a) on Earth and $(b, c)$ in space. In the presence of gravity, all tubes grow upward driven by buoyancy. In the absence of gravity, we see tubes growing at angles from each other.

silicate concentrations for all of the studied salts. Nevertheless, the microgravity experiments in $0.6 \mathrm{M}$ produce a slightly larger osmotic envelope than in ground experiments. This is observed in calcium, manganese, and cobalt gardens (Figure 2).

Under our experimental conditions, $\mathrm{MnCl}_{2}$ gardens have a lower induction time than $\mathrm{CaCl}_{2}$ gardens. However, at the end of growth the number of tubes is higher with calcium than with manganese. When manganese gardens are grown on Earth, they usually develop the wide, twisted tubes that we can see in the $6 \mathrm{M}$ silicate example in Figure 2. We did not observe this type of tube formation in microgravity. In the $2 \mathrm{M}$ silicate flight experiment, the manganese salt started to dissolve as soon as it was introduced into the chamber, producing microscopic tubes growing in all directions (Figure 2). The main tube grew more rapidly than in the case of the other salts, at approximately $1 \mathrm{~cm}$ per minute, even after bouncing off the chamber wall. Only narrow tubes were formed in on-ground growth in $0.6 \mathrm{M}$ silicate according our previous studies. ${ }^{6}$ In microgravity, tubes failed to grow initially in the $0.6 \mathrm{M}$ silicate solution, but after 4 days, a very thin tube appeared growing in a non-upward direction (Figure 2).

We can see the open section of manganese tubes grown on Earth and in space in Figure 5. The images taken with a backscattered electron detector show us how the inner and outer sides of the tube wall are formed of different materials: manganese oxide on the inside (bright in the figure) comprising the main thickness of the wall and manganese silicate (dark in the figure) covering the outside. In general, we observe that the gradient between the external metal silicate species and the internal metal oxide-hydroxide species is sharper in Earth than in space growths. Probably, the mass transport and precipitation kinetics are different under microgravity, altering this compositional gradient. 

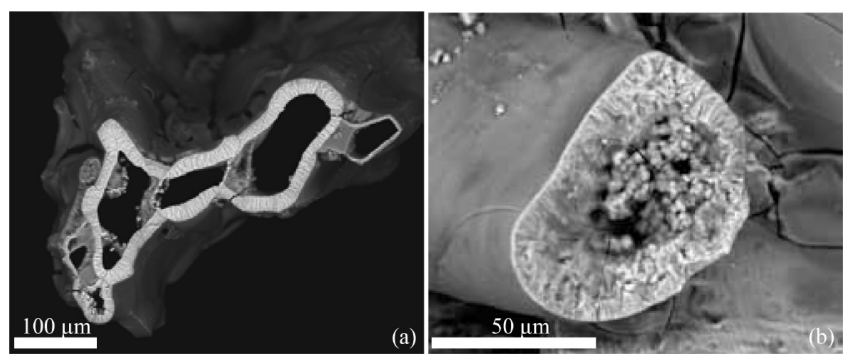

Figure 5. Micrographs of manganese tubes grown in $2 \mathrm{M}$ sodium silicate taken with a backscattered electron detector; the brighter areas are made of manganese oxide and the darker of manganese silicate: (a) on Earth, (b) in microgravity.

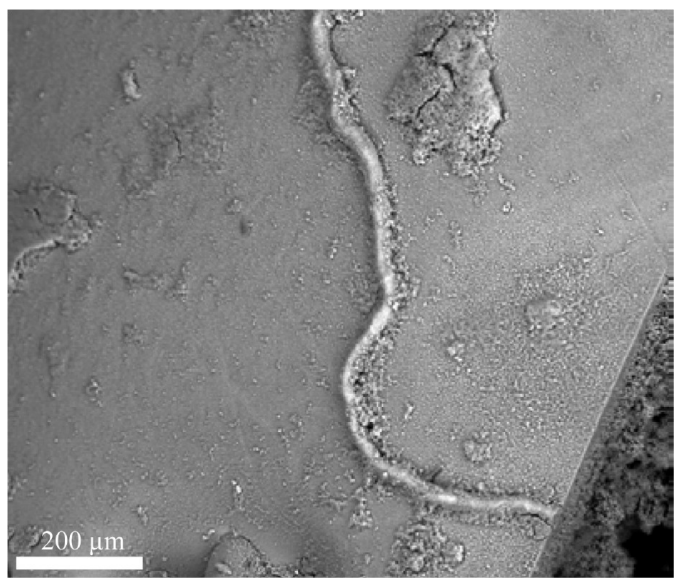

Figure 6. Micrograph of a possible manganese tube growing along the external surface of the osmotic envelope of the initial salt in $0.6 \mathrm{M}$ sodium silicate in microgravity.

SEM micrographs and EDX analysis on a section of the wall of the osmotic envelope of manganese gardens grown in $0.6 \mathrm{M}$ silicate under microgravity confirmed that it was completely composed of manganese silicate, but we found a surface vein that was richer in manganese oxide. This may have been a tube growing along the external surface of the sphere (Figure 6).

With cobalt, an upward growth of narrow blue tubes is observed in Earth gravity in 6 and $2 \mathrm{M}$ silicate, being faster in 1 and $0.6 \mathrm{M}$. In microgravity, however, there was active growth at $2 \mathrm{M}$, forming narrow tubes in random directions with an average growth rate of $0.3 \mathrm{~cm}$ per minute, but no significant tube growth was observed at 1 and $0.6 \mathrm{M}$.

The SEM image in Figure 7a shows the beginning of tube growth on the surface of the osmotic envelope in an experiment with $\mathrm{CoCl}_{2}$ in $2 \mathrm{M}$ silicate in microgravity. We can see that the direction of those tubes is random instead of only upward. In Figure $7 b$, we can see the inner surface of a tube from the same experiment. The surface is covered by rosettes of crystals composed of cobalt oxide-hydroxide. This morphology has also been detected on Earth ${ }^{6}$ and in other cobalt oxides. ${ }^{10}$

In the case of nickel, experiments performed on Earth produced shorter tubes than the other salts, with a growth maximum at $0.6 \mathrm{M}$ according to our previous studies. ${ }^{6}$ Experiments in microgravity showed that $\mathrm{NiSO}_{4}$ gardens grown in microgravity exhibited lower growth velocities than the other salts, at $0.1 \mathrm{~cm}$ per minute. The most reactive silicate concentration was $2 \mathrm{M}$, producing a few short tubes in random directions.

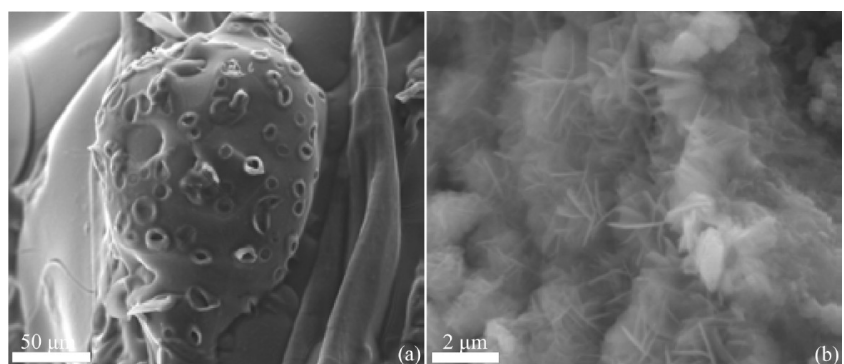

Figure 7. Micrographs of $\mathrm{CoCl}_{2}$ growing in $2 \mathrm{M}$ silicate in microgravity: (a) the beginnings of several tubes from the osmotic envelope follow random directions; (b) on the inner surface of a tube, we can see rosettes of cobalt oxide-hydroxide crystals.

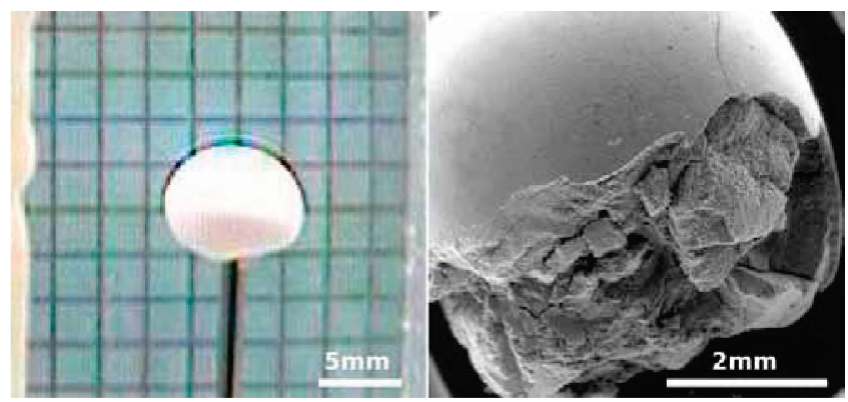

Figure 8. (a) Photograph of $\mathrm{CaCl}_{2}$ experiment performed in space with $0.6 \mathrm{M}$ sodium silicate. (b) SEM micrograph of this osmotic envelope at the end of experiment.

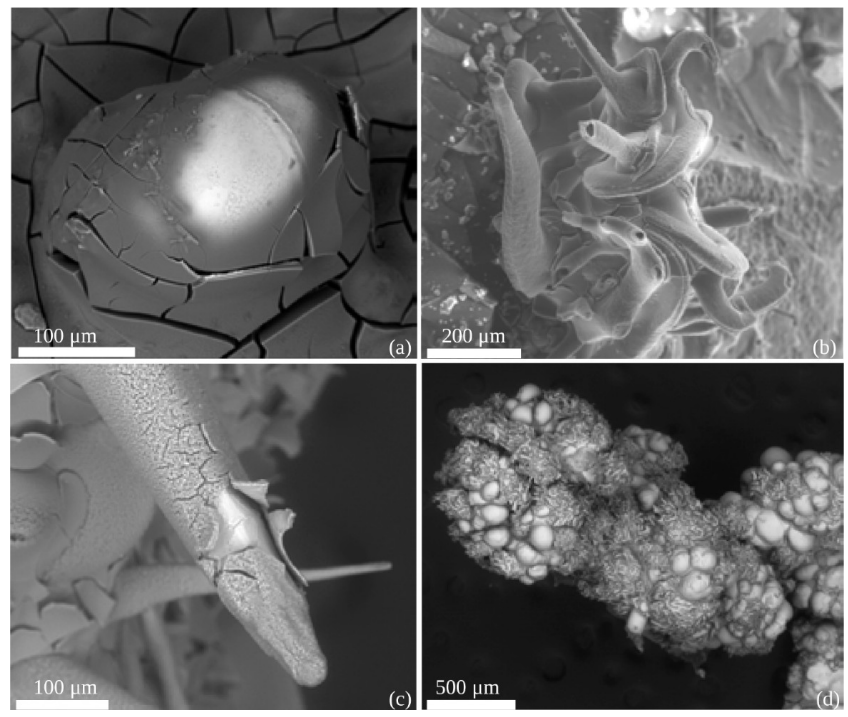

Figure 9. SEM micrographs of examples of plastic deformation in microgravity (a, c, and d were taken with a backscattered electrons detector): (a) $\mathrm{MnCl}_{2}$ in $6 \mathrm{M}$ silicate. The brighter region is rich in $\mathrm{MnO}$ and the darker in $\mathrm{MnSiO}_{3}$. (b) $\mathrm{NiSO}_{4}$ in $2 \mathrm{M}$ silicate. Several twisted fingers grown by plastic deformation. (c) $\mathrm{NiSO}_{4}$ in $2 \mathrm{M}$ silicate, the external dark layer is $\mathrm{SiOn}$ and the bright internal layer is nickel silicate. (d) Highly twisted tube of $\mathrm{NiSO}_{4}$ in $1 \mathrm{M}$ silicate grown on Earth. In the presence of gravity, we did not see plastic deformation.

Other experiments, not shown here, did not produce results owing to several apparatus failures, such as having air bubbles trapped in the 

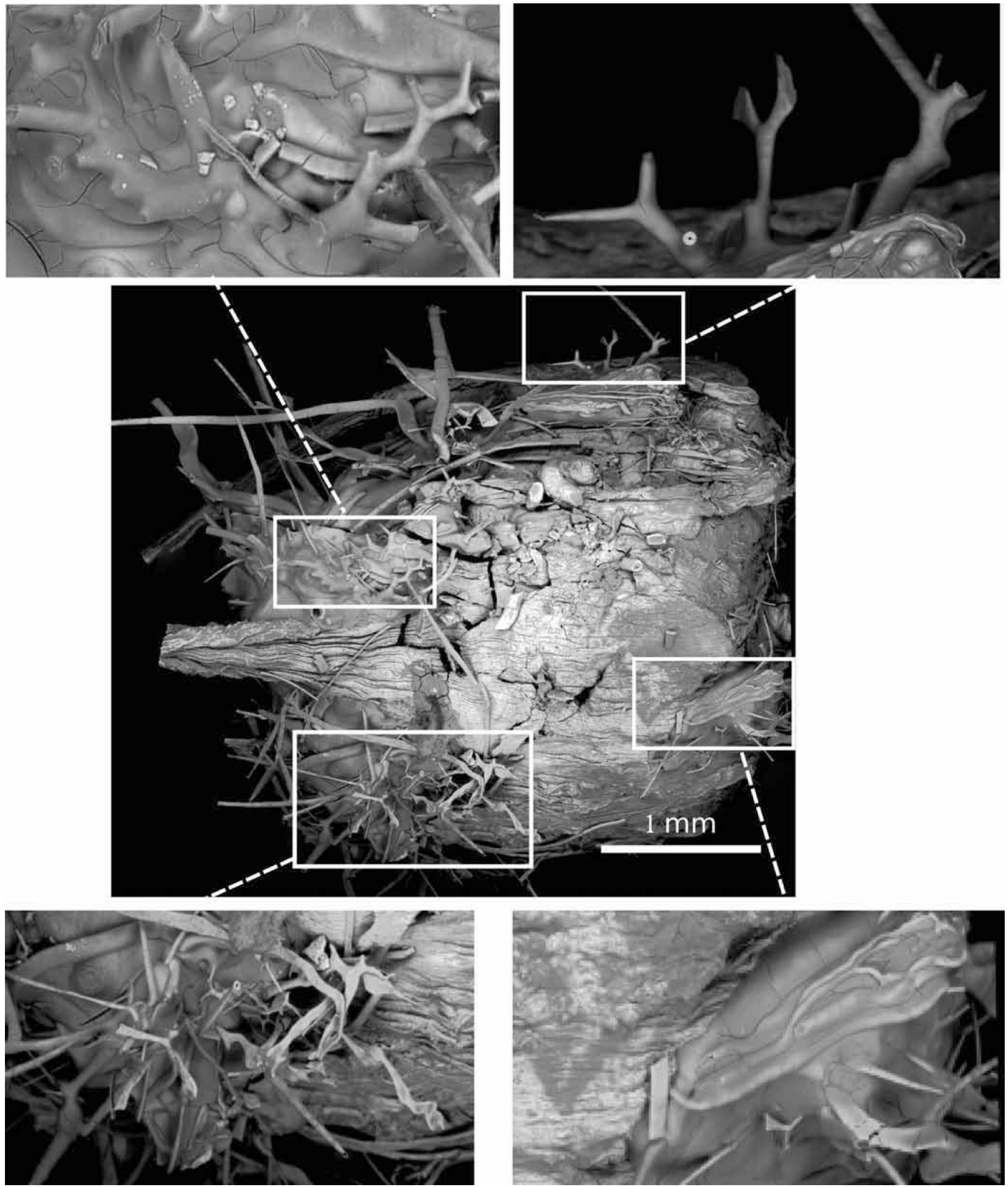

Figure 10. General view of a garden grown from $\mathrm{MnCl}_{2}$ in $2 \mathrm{M}$ sodium silicate in microgravity. In this image, we see different shapes and textures corresponding to the different growth modes. Most of the longer tubes were broken during transportation. Insets show close-ups on plastic and branched growth. The salt entered the reactor chamber from left to right as seen on this picture.

reactor chamber or the jamming of the push rods. Some experiments did not have enough time to complete owing to time limitations in the International Space Station and the slower growth in microgravity.

Besides the different growth-orientation behavior, the most evident difference between the two sets of experiments, on ground and in space, was the growth rate; it took several days to accomplish in space what only takes an hour on Earth.
Growth Rate. Chemical gardens grow by the reaction of cations flowing out from the inner solution, after breaking the membrane, precipitating the silicate of this cation with the external solution. After forming this wall, the interaction of the cation, solvated in a basic solution, from the inner solution with the internal face of the wall produces the precipitation of the cation oxide-hydroxide on the internal face of the wall. ${ }^{4}$ The 
precipitation involves depletion of reactive molecules around the growth area, creating a concentration gradient. In the presence of gravity, this gradient is automatically and rapidly reduced by convection. In space, however, the depletion of reagents owing to the suppression of buoyancy-driven convection reduces the growth rate, with the reaction becoming diffusion-limited. ${ }^{11}$ In this manner, a reaction that takes a few minutes to complete in Earth gravity can last for several days in space. In the calcium chloride example shown in Figure 2, the Earth experiment grew at $2 \mathrm{~cm}$ per second, while the space tubes grew at less than $1 \mathrm{~mm}$ per hour. Other salts exhibited similarly large differences in growth rates between Earth gravity and microgravity experiments.

A sequence of simultaneous growth of chemical gardens for the different salts on Earth is described in the Supporting Information (SI). The cobalt and manganese salts are the first to start growing small, short tubes. When the growth of these salts is almost finished, the calcium salt starts growing at a considerable rate. On the contrary, nickel salts have a short induction time but produce short tubes with the lowest growth rate. The same relative differences was observed in microgravity. However, in some extreme cases, where the silicate concentration was not optimal for growth, no tubes at all appeared under microgravity. Instead, a membrane envelopes the initial salt swelling with water by osmosis and inflating up to several times its initial volume, but neither bursting nor growing any tubes. Upon SEM examination of these osmotic envelopes, we found no discernible morphology differences across their section and the chemical composition was essentially metal silicate, with increasing metal concentration toward the interior of the envelope. No compositional gradient was observed, and no crystals of metal oxide-hydroxide were detected in the osmotic envelopes, whereas on the contrary, they were detected in the interior of the tubes; see Figure 8.

Plastic Deformation. The slow growth in the absence of gravity also accounted for a different type of growth that Jones and Walter named "plastic deformation", 7 as opposed to "openended tubes". The shortage of precipitant reagents owing to the absence of density-driven convection results in the initial semipermeable membrane remaining fluid for a relatively long time. Any excess of pressure in some region of the membrane would be expected to make it bulge outward, forming a hump on the membrane surface. We show an example of just this situation in a manganese garden grown in $6 \mathrm{M}$ silicate (Figure 9a), where the surface of the membrane has bulged by inner pressure without bursting. The brighter region is rich in $\mathrm{MnO}$ and the darker in $\mathrm{MnSiO}_{3}$. The cracks on the surface appeared during the drying of the sample. As the membrane continued to push outward, its walls would become thinner and more flexible, presenting less resistance to the pressure inside and forming an elongated shape like a plastic finger. This can be described as a Laplacian growth instability, as was noted by Mullins and Sekerka. ${ }^{12}$ We can see examples in a nickel garden grown in space with $2 \mathrm{M}$ silicate, where fingers grow in different random directions with a smooth and continuous morphology (Figure 9b,c). Eventually, silicate precipitates from the outside and metal oxide-hydroxide from the inside, hardening and preserving the structure. This is a growth mechanism not observed in Earth experiments, which produce very irregular shapes, not always straight and not always cylindrical in cross section, like the highly twisted tube of Figure 9d, with a very complex surface. On Earth, the buoyancy forces of the inner solution change the growth mechanism.

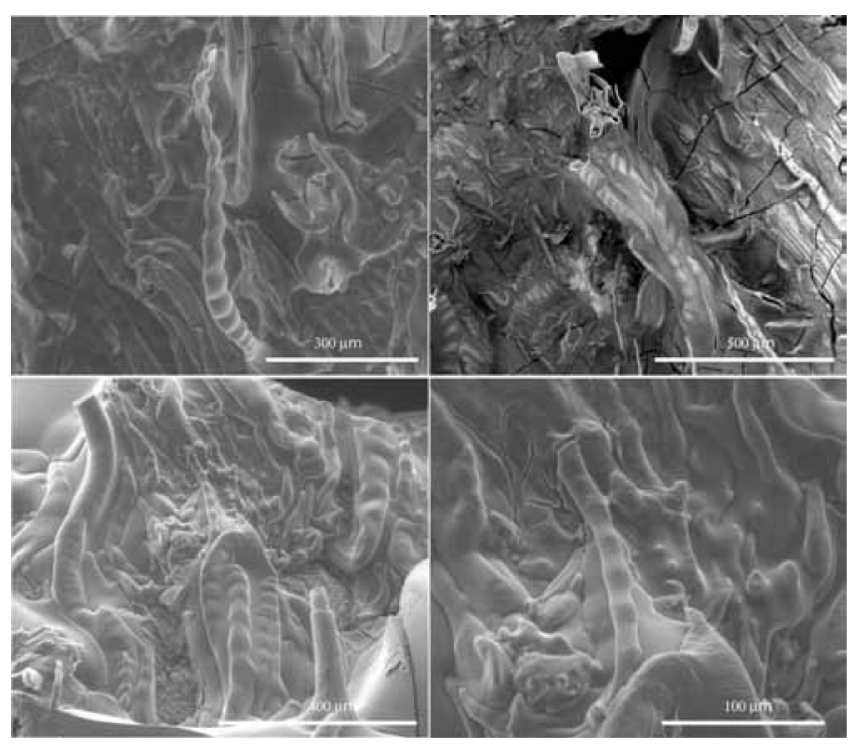

Figure 11. Examples of oscillatory growth in experiments performed on Earth (first row) and in microgravity (second row). The experimental conditions were $\mathrm{CoCl}_{2}$ in $2 \mathrm{M}$ silicate.

Instead of plastic deformation, buoyancy breaks the nickel silicate wall anywhere, forming a cluster of many tubes that are blocked again by the precipitation of nickel silicate following which the blockage is broken again in another direction and so on, forming a highly irregular surface where many lateral twisted microtubes and spheroidal bubbles are observed. The chemical analysis of this surface shows a higher content of metal oxide-hydroxide in the brighter bubble zones than in the darker microtube zones.

In Figure 10, we show an SEM image of a $\mathrm{MnCl}_{2}$ garden grown in $2 \mathrm{M}$ sodium silicate in microgravity. This particular experiment has instances of both the classic, chemical garden tubular growth and the microgravity exclusive plastic growth. The random orientation of the tubes is clearly observed. Some tubes grow attached to the surface, whereas others grow separately. Some tubes are completely straight, while others are twisted. Some peculiar branching patterns of these tubes are observed forming $120^{\circ}$ angles alternating consecutively along the tube. These patterns have not been reported previously in chemical gardens, and we cannot explain them, although branched tube growth was suggested by Cartwright et al. ${ }^{1}$

Oscillatory Growth. Chemical gardens on Earth have been reported to grow by pulses due to relaxation oscillations of the silicate membrane. ${ }^{13^{-15}}$ The resulting tubes look sectored or periodically constricted. This growth regime can be provoked by injecting the metal salt already dissolved at a specific concentration and pressure; Thouvenel-Romans et al. ${ }^{13}$ found that tubes could switch from steady to pulsating growth modes by changing the concentration of the injected metal salt solution. The same research group proved that tube growth can be templated by buoyant gas bubbles ${ }^{15}$ at the tip of the growing tubes and, in these cases, that the external surface of the tubes is patterned with silicate rings, a phenomenon that we have also observed even in the absence of gravity. The bubble at the tube tip dictates the tube's radius and also influences the growth rate by volume conservation of the injected solution. We observed this oscillatory form of tube both on Earth and in space. In Figure 11, we show examples of oscillatory growth of a cobalt garden in $2 \mathrm{M}$ silicate on Earth $(\mathrm{a}, \mathrm{b})$ and in space $(\mathrm{c}, \mathrm{d})$. The sequence 
of these oscillations seems to be regular (i.e., each $40 \mu \mathrm{m}$ linearly along the tube). Most of the tubes grow attached to the surface; one tube grown separately was observed in Earth experiments.

\section{CONCLUSIONS}

The purpose of these experiments was to remove the influence of buoyancy during the formation of chemical gardens to focus on the forced convection part of the phenomenon. We expected to obtain straight tubes emanating from the initial salts, and indeed, in some cases such tubes were observed. When the silicate membrane does break, it does so in any given direction and forms a tube that will grow along that initial direction until the metal solution is depleted or until it reaches the chamber wall, where it may even reflect off the wall and continue to grow in a new direction.

The slower growth exerts an extremely strong influence, something reported as well by Jones and Walter in their space experiments. ${ }^{7}$ The absence of gravity means there is no convection due to buoyancy. A diffusive zone of reagent depletion becomes then an important factor, slowing down the reaction from hours to days and establishing a diffusion-limited environment. ${ }^{11}$ The duration of this lengthened process was not adequately taken into account in scheduling time allocated for the experiment on the International Space Station.

The reduced growth rate also had an apparent secondary effect, the appearance of a totally different growth regime. Plastic deformation by the swelling of the membrane produces morphologies that are not seen on Earth, introducing new questions about the malleability of these materials and their hardening process. We also found a remarkable abundance of sectored or periodically constricted tubes, which was surprising but may perhaps be attributed to the diffusion-limited growth regime, which reduces the flow of the metal-ion salt solution.

Some of the morphologies that have been detected under microgravity are biomimetic, although they are of course abiotic. This can be a warning to extraterrestrial life researchers that apparently biological morphologies can have a nonbiological origin. ${ }^{16}$

The chemical composition of silicate gardens grown in space did not differ from those grown under Earth gravity. We still found metal oxides on the inside of tubes and metal silicates on the outside. Only a few crystalline phases could be identified using powder diffraction, which is similar to what other studies on Earth have demonstrated. Nevertheless, the compositional gradient between metal oxide-hydroxide and metal silicate phases observed on Earth was not clearly observed under microgravity. In spite of the difficulties to prepare experiments in space, further experiments should be perfomed in order to find further explanations of all phenomena observed in this work.

\section{ASSOCIATED CONTENT}

S Supporting Information. Some figures have been included. This material is available free of charge via the Internet at http://pubs.acs.org.

\section{AUTHOR INFORMATION}

\section{Corresponding Author}

*E-mail: julyan.cartwright@csic.es; bruno.escribano.salazar@gmail. com; ignacio.sainz@iact.ugr-csic.es; stodieck@colorado.edu.

\section{Present Addresses}

${ }^{\S}$ Basque Center for Applied Mathematics (BCAM), Building 500 Bizkaia Technology Park, E-48160 Derio, Spain.

\section{ACKNOWLEDGMENT}

We dedicate this work to the crew of the Space Shuttle Columbia, who died as it disintegrated on its return from orbit on February first 2003; Columbia carried onboard an SGHab with similar experiments to those presented here. We thank those at BioServe and at the National Aeronautics and Space Administration (NASA), including the astronauts, who made these experiments possible and saw to their execution onboard the ISS. We should also like to thank our colleagues at Orion's Quest who developed a course of study involving similar experiments to those here that was used by numerous students in their classrooms as they participated in this flight research.

\section{REFERENCES}

(1) Cartwright, J. H. E.; García-Ruiz, J. M.; Novella, M. L.; Otálora, F. J. Colloid Interface Sci. 2002, 256, 351-359.

(2) Hazlehurst, T. J. Chem. Educ. 1941, 18, 286-289.

(3) Collins, C.; Zhou, W.; Mackay, A.; Klinowski, J. Chem. Phys. Lett. 1998, 286, 88-92.

(4) Coatman, R. D.; Thomas, N. L.; Double, D. D. J. Mater. Sci. 1980, $15,2017-2026$.

(5) Cartwright, J. H. E.; Escribano, B.; Khokhlov, S.; Sainz-Díaz, C. I. Phys. Chem. Chem. Phys. 2011, 13, 1030-1036.

(6) Cartwright, J. H. E.; Escribano, B.; Sainz-Díaz, C. I. Langmuir 2010; DOI: $10.1021 /$ la104192y.

(7) Jones, D. E. H.; Walter, U. J. Colloid Interface Sci. 1998, 203, 286 293.

(8) Stockwell, B.; Williams, A. School Sci. Rev. 1994, 76, 7-14.

(9) Orions Quest, http://www.orionsquest.org.

(10) Tao, Y.; Zhu, B.; Chen, Z. J. Cryst. Growth 2006, 293, 382-386.

(11) Carotenuto, L.; Cartwright, J. H. E.; Castagnolo, D.; García-

Ruiz, J. M.; Otálora, F. Micrograv. Sci. Technol. 2002, 13, 14-21.

(12) Mullins, W. W.; Sekerka, R. F. J. Appl. Phys. 1964, 35, 444-451.

(13) Thouvenel-Romans, S.; Steinbock, O. J. Am. Chem. Soc. 2003, $125,4338-4341$.

(14) Thouvenel-Romans, S.; van Saarloos, W.; Steinbock, O. Europhys. Lett. 2004, 67, 42-48.

(15) Thouvenel-Romans, S.; Pagano, J.; Steinbock, O. Phys. Chem. Chem. Phys. 2005, 7, 2610-2615.

(16) Livage, J. C. R. Palevol. 2009, 8, 629-636. 